\title{
QUALIDADE E DINÂMICA VOCAL DE UM FALANTE HOMOSSEXUAL À LUZ DO VOCAL PROFILE ANALYSIS SCHEME FOR BRAZILIAN PORTUGUESE-VPAS- PB: UM RELATO DE CASO
}

\section{QUALITY AND VOCAL DYNAMICS OF A HOMOSEXUAL SPEAKER IN THE LIGHT OF THE VOCAL PROFILE ANALYSIS SCHEME FOR BRAZILIAN PORTUGUESE- VPAS-PB: A CASE REPORT}

\author{
Rogério Marcelino dos Santos Melo ${ }^{54}$ \\ Eduardo Lucas Sousa Enéas ${ }^{55}$ \\ Ericles Ferreira Valões ${ }^{56}$ \\ Maria Fabiana Bonfim de Lima Silva ${ }^{57}$
}

\begin{abstract}
RESUMO: Os estudos em linguagem permitem compreender novas dimensões sociais e individuais, por meio da aplicação de diferentes técnicas investigativas sobre o mesmo objeto de estudo. As investigações sobre os aspectos perceptivo-auditivos auxiliam na compreensão dos ajustes do trato vocal, de tensão muscular e fonatórios de um sujeito (CAMARGO e MADUREIRA, 2008). Este trabalho tem como objetivo descrever a qualidade e dinâmica vocal de um falante homossexual do sexo masculino em dois momentos, antes e depois de assumir sua homossexualidade, por meio do roteiro Vocal Profile Analysis Scheme for Brazilian Portuguese $V P A S-P B$. A pesquisa do tipo qualitativa é baseada em relato de caso, sendo composta por duas coletas de fala semiespontânea desse sujeito homossexual. As amostras foram obtidas em ambiente acusticamente tratado com intervalo de gravação de três anos; posteriormente as amostras foram avaliadas por um juiz com o uso do roteiro $V P A S-P B$. Os resultados mostram que o participante, após os três anos, apresentou mudanças: de corpo de língua abaixado para avançado; expansão faríngea para constrição faríngea; de laringe abaixada para laringe elevada; de modal para falsete (intermitente); e o aumento da graduação dos ajustes de mandíbula fechada e hiperfunção do trato vocal. No que se refere aos aspectos de dinâmica vocal: pitch habitual abaixado para elevado; extensão e variabilidade de pitch de diminuído para aumentado, bem como aumentou a graduação da taxa de elocução. Além disso, foi identificado na voz do falante estudado ajustes de lábios estirados e hiperfunção laríngea. Nessa investigação foi possível traçar o perfil vocal do falante homossexual em momentos distintos identificando ajustes marcantes na fala deste sujeito, bem como as relações sociais e de atribuições de significados estabelecidos por esses ajustes.
\end{abstract}

Palavras-chave: Homossexual. Linguística. Fonética.

\begin{abstract}
Language studies allow us to understand new social and individual dimensions by applying different investigative techniques on the same object of study. Investigations on auditory-perceptual aspects help us to understand the vocal tract, muscle tension and phonatory adjustments of a subject (CAMARGO and MADUREIRA, 2008). This paper aims to describe the vocal quality and dynamics of a homosexual male speaker in two moments, before and after coming out about his sexuality, through the script Vocal Profile Analysis Scheme for Brazilian Portuguese - VPAS-PB. The qualitative research is based on a case report, consisting of two semi-spontaneous speech collections of a homosexual subject. The samples were obtained in an acoustically treated environment with a three-year recording interval; then, the samples were evaluated by a judge using the VPAS-PB script. The results show that the participant, after the three-year interval, presented changes, ranging from a lowered tongue body to an advanced one; pharyngeal expansion to pharyngeal constriction; lowered larynx to an elevated one; from modal to falsetto (intermittent); and increased graduation of closed jaw adjustments and vocal tract hyperfunction. Concerning vocal dynamics aspects: usual lowered
\end{abstract}

\footnotetext{
${ }^{54}$ Mestrando do Programa de Pós-Graduação em Linguística da Universidade Federal da Paraíba (UFPB). Email: rogeriomarcelino.letras2013@gmail.com.

${ }^{55}$ Mestrando do Programa de Pós-Graduação em Linguística da Universidade Federal da Paraíba (UFPB). Email: eduardolucas.vip@hotmail.com.

${ }_{56}$ Graduado em Fonoaudiologia pela Universidade Federal da Paraíba (UFPB). E-mail: ericlesferreiravaloes@gmail.com.

${ }^{57}$ Docente do Departamento de Fonoaudiologia, do Programa de Pós-Graduação em Linguística e do Programa de Pós-Graduação em Fonoaudiologia da Universidade Federal da Paraíba (UFPB). E-mail: fbl_fono@yahoo.com.br.
} 
pitch to high; extension and pitch variability from decreased to increased as well as increased graduation in speech rate. In addition, the speaker's voice was identified with stretched lips adjustments and laryngeal hyperfunction. In this investigation, it was possible to trace the homosexual speaker's vocal profile at different moments, identifying marked adjustments in the speech of this subject as well as the social relations and attributions of meanings established by these adjustments.

Keywords: Homosexual. Linguistics. Phonetics.

\section{Introdução}

A linguística tem demonstrado seu caráter interdisciplinar nas últimas pesquisas, a exemplo das investigações sociolinguísticas com enfoque as propriedades fonéticas dos falantes da língua. A caracterização dos diferentes modos de articular e produzir os sons de uma língua pelos falantes permite constatar as variações e mudanças da língua condicionadas por fatores sociais, estilísticos e avaliativos (LABOV,1978).

Os estudos linguísticos têm aplicado diferentes abordagens investigativas para explicar as diferentes dimensões do seu objeto de estudo, a linguagem, a exemplo da adoção de técnicas fonéticas ancoradas aos fatores socais. Nesse sentido, observar a dinâmica vocal é adotar como ponto de análise as propriedades da voz e da fala dentro de uma integração nas esferas perceptivo-auditiva, acústica e fisiológica na qual compreende-se essas características como agentes fundamentais no processo comunicativo do falante. (CAMARGO e MADUREIRA, 2008).

Dentro da esfera perceptivo-auditiva, o modelo fonético de descrição da qualidade vocal, criado por Laver (1980), vislumbra a qualidade vocal como resultado das diferentes movimentações musculares possíveis pelo trato vocal: laríngeo (ajustes fonatórios), supralaríngeo (ajustes articulatórios) e de tensão muscular (ajustes de tensão). Logo, a caracterização da fala a partir do sinal vocal deve ser analisada pelos ajustes parciais ou totais produzidos pelos falantes (CAMARGO, 2002).

Nesse sentido, partindo desse modelo fonético, Laver et al. (1981) elaboraram um roteiro de avaliação perceptivo-auditiva da qualidade e dinâmica vocal denominado Vocal Profile Analysis Scheme - VPAS. O avaliador constrói, por meio do VPAS, um perfil vocal do que escuta na fala de um indivíduo de forma recorrente (LAVER et al., 1981; LAVER, 2000; LAVER e MACKENZIE-BECK, 2007).

É nessa linha de raciocínio que este trabalho se insere, sendo o objetivo descrever a qualidade e dinâmica vocal de um falante do sexo masculino em dois momentos - antes e depois de se assumir homossexual por meio do roteiro VPAS-PB (CAMARGO e MADUREIRA, 2008). Ressalta-se que os dois momentos distintos que motivaram essa descrição (da qualidade e dinâmica vocal) desse falante estão separados por um intervalo de tempo de três anos entre as coletas.

A discussão dos resultados fonéticos obtidos foi baseada no modelo fonético de descrição da qualidade vocal (Laver, 1980) e na terceira onda da Sociolinguística focalizando o falante, numa busca por compreender que significados sociais esses falantes atribuem às variantes e como os querem transmitir e por qual motivo (CEZÁRIO e VOTRE, 2011; ECKERT, 2012).

No que se refere aos trabalhos fundamentados na abordagem fonética utilizando o $V P A S$ - PB, os estudos ainda são escassos na região nordeste do Brasil, destacando-se nesse território as pesquisas de Lima et al. (2007) e de Medeiros (2019). Por outro lado, ainda não há trabalhos com o $V P A S$ - PB com sujeitos homossexuais em diferentes regiões, reforçando a necessidade de se pesquisar o modo de falar deste grupo em diferentes contextos.

Por isso, foi desenvolvido o presente estudo, que considera a qualidade vocal de sujeitos homossexuais, por acreditar na suma relevância destes achados para o campo da Linguística, 
no tocante às representações da língua, quanto para a Fonoaudiologia, no que se refere às questões vocais, auxiliando no desenvolvimento de métodos para o atendimento a esse público específico.

\section{Fundamentação teórica}

A teoria Sociolinguística considera uma língua natural como uma complexa cadeia de fenômenos passíveis de variação das mais diversas escalas. O escopo da Sociolinguística se destina a estudar as relações entre a estrutura linguística e as condições sociais e culturais envolvidas na sua produção (CEZÁRIO; VOTRE, 2011). Dessa forma, a língua não pode ser estudada como uma estrutura autônoma, sem levar em consideração a história, a cultura e as diversas facetas sociais que se manifestam na sua produção. Ressalta-se que a Teoria Sociolinguística surge como uma refutação à ideia de que a fala e suas variações eram livres e desordenadas, feita pelo Linguista Willian Labov ([1978] 2008) - numa crítica direta ao posicionamento bloomfieldiano acerca das variações e do tratamento da fala. Labov mostrou ser possível e plausível estudar e ordenar o "caos linguístico" em níveis distintos, que tinham como plano de fundo a conjuntura social.

$\mathrm{Na}$ atualidade, os estudos da Sociolinguística podem ser realizados sob o prisma de três ondas, propostas por Penelope Eckert (2012) após análises dos trabalhos realizados na área. A proposta da autora se volta para uma (re)definição do campo em três ondas, na quais as formas de compreender a produção vernacular diferem entre si, porém não assumem caráter de exclusão ou substituição uma sobre as outras (NASCIMENTO, 2017).

Na primeira onda da Sociolinguística, o foco de análise se volta para o desenvolvimento do grande quadro, por assim dizer, no qual as pesquisas (mais tradicionais) se voltam para a descrição das variantes linguísticas em correlação com categorias primárias de estratificação, como sexo, idade, fatores socioeconômicos. A segunda onda, por seu turno, traz pesquisas cujo foco de análise reflete categorias sociais localmente definidas, apresentando as significações sociais locais para as variantes linguísticas (ECKERT, 2012).

Finalmente, a terceira onda da Sociolinguística vislumbra de modo ligeiramente diferente a significação social das variantes - macro ou micro - porque considera que essa significação não pode ser estudada fora de contextos e situações reais de uso, atestando que um dado significado social pode variar de uma situação para outra, a depender do interlocutor e do contexto comunicativo. Assim, a terceira onda se focaliza mais precisamente no falante, numa busca por compreender que significados sociais esses falantes atribuem às variantes e como os querem transmitir e por qual motivo. Além disso, duas nuances da terceira onda se destacam com acentuação: estilo e comunidades de práticas. O estilo, de acordo com Eckert (2012), reflete uma forma de interação social na qual há uma intenção reflexiva no ato de comunicar que legitima as escolhas do falante sobre seu estilo na interação em grupos sociais. Acerca das comunidades de práticas, pode-se afirmar que estas tomam forma na relação estabelecida entre seus participantes a partir das práticas comuns vivenciadas entre si que os fazem interagir de forma particular, a exemplo da família, amigos, colegas de trabalho. De tal forma, essas práticas tomam corpo influenciando no compartilhamento da visão social de seus participantes.

Concebendo que a dicotomia entre voz e fala deve ser deixada de lado, acredita-se que os estudos sobre a voz também podem se relacionar nos estudos da Sociolinguística à medida em que as manifestações de uso na qualidade vocal refletem uma gama de problemáticas passíveis de análise sob o viés Sociolinguístico.

Levando em consideração que a voz é uma importante ferramenta social e que por meio dela pode-se transmitir características emocionais, físicas e de personalidade, acredita-se que 
o falante homossexual pode apresentar uma tendência a realizar ajustes no aparelho fonador que sejam compatíveis com os significados sociais por ele atribuído, e em relação às suas comunidades de prática. Desta forma, o presente estudo também considera como referencial teórico a abordagem fonética de descrição da qualidade vocal de autoria de Laver (1980), que parte de uma unidade de análise o setting (ajuste).

Esse autor explica que, de acordo com o modelo fonético, a qualidade vocal é considerada como um agrupamento de ajustes marcantes, com mobilizações nos planos laríngeo (ajustes fonatórios), supralaríngeo (ajustes articulatórios) e de tensão muscular (ajustes de tensão) do aparelho fonador que caracteriza a fala de um indivíduo de maneira parcial ou total. Sendo assim, a qualidade vocal resulta de ajustes fonatórios, articulatórios e de tensão.

O modelo fonético que descreve a qualidade vocal é baseado em pistas auditivas, acústicas, fisiológicas e articulatórias de vastas mobilizações do aparelho fonador durante a realização emissão sonora (LAVER, 1980).

A qualidade vocal destaca-se por sua grande importância nas interações sociais, tanto em termos comunicativos quanto informativos e é o produto da combinação do falante em seu estado orgânico (fatores intrínsecos) e os ajustes fonéticos realizados (fatores extrínsecos) pelo aparelho fonador. São estes últimos conhecidos como ajustes musculares de longo termo, que são de controle voluntário, dispostos conforme questões culturais, linguísticas, paralinguísticas e utilizadas por falantes de forma comunicativa. Pode-se afirmar que são controláveis os ajustes musculares, podendo ser desenvolvidos, aprendidos e modificados, caso necessário (MACKENZIE-BECK, 2005).

Vários estudos foram realizados sob a perspectiva fonética no Brasil. Os autores apontaram vantagens nesta abordagem da qualidade vocal (MADUREIRA, 1992; SPINA E CRISPIM, 1999; LIMA ET AL., 2007; CAMARGO E MADUREIRA, 2009; LIMA, MADUREIRA E CAMARGO, 2009; FERNANDES, 2011). Madureira (2008) afirmou que a Fonética, além de promover o aperfeiçoamento da escuta, permite a integração de informações sobre produção e a percepção, permitindo a análise mais detalhada da qualidade vocal.

A partir deste modelo foi elaborado o roteiro de avaliação perceptivo-auditiva da qualidade vocal, Vocal Profile Analysis Scheme- VPAS (LAVER et al, 1981), que recentemente foi adaptado para o português brasileiro $V P A S-P B$ (CAMARGO e MADUREIRA, 2008). Tal roteiro detalha a ocorrência de diversos ajustes, nos planos articulatório, fonatório e de tensão, bem como elementos da dinâmica vocal (pitch, loudness, duração e suporte respiratório), sob a perspectiva fonética. O VPAS-PB é uma ferramenta que tem como finalidade descrever as características mais marcantes na qualidade vocal e nos aspectos de dinâmica vocal. Dessa forma, o avaliador constrói por meio deste instrumento um perfil vocal do que escuta na fala de um indivíduo de forma recorrente (LAVER et al., 1981; LAVER, 2000; LAVER e MACKENZIE-BECK, 2007).

Portanto, o presente estudo tem respaldo nestas duas abordagens (Fonética e Sociolinguística) que fundamentaram a análise desse estudo de caso.

\section{Metodologia}

A pesquisa de caráter qualitativo, longitudinal e observacional buscou descrever a qualidade vocal de um falante homossexual, por meio do roteiro $V P A S-\mathrm{PB}$, em dois períodos distintos separados por um intervalo de tempo de três anos entre as coletas (2016 e 2019).

A coleta de voz ocorreu em um estúdio profissional, utilizando-se o software Sound Forge versão 10.0, desktop Dell All-In-On, microfone cardioide unidirecional, da marca Senheiser, modelo E-835, localizado em um pedestal e acoplado a um pré-amplificador Behringer, modelo UPhoria UMC 204. 
A voz foi coletada em cabine de gravação com tratamento acústico e ruído inferior a 50 dB NPS, com taxa de amostragem de $44000 \mathrm{~Hz}$, com 16 bits por amostra e distância de $10 \mathrm{~cm}$ entre microfone e a boca do participante. Para a coleta da voz, o sujeito estava em pé, situando-se $\mathrm{o}$ pedestal à sua frente, de acordo com a distância preconizada entre a boca e o microfone. $\mathrm{O}$ sujeito recebeu as instruções sobre a coleta de voz e, logo em seguida, precedeu-se com o registro.

O procedimento de gravação correspondeu a um trecho de fala semiespontânea que foi retirado de uma entrevista e de uma situação de diálogo estabelecido entre o sujeito da pesquisa e outro interlocutor, no qual foram utilizadas fotos de personalidades da área política e artística para facilitar e conduzir a conversa entre si.

Nos dois momentos da coleta (primeiro momento em 2016 e o segundo em 2019), foram realizadas entrevistas semiestruturadas relacionadas à identificação pessoal: nome, idade, local de nascimento e de residência atual, sexo, gênero, escolaridade, trabalho e uso de medicamentos e/ou hormônios.

Em 2016, o participante relatou que tinha 18 anos, nasceu em Pernambuco, residia em João Pessoa, sexo masculino, não afirmava ser homossexual, tinha ensino médio completo, cursando o primeiro período de um curso de nível superior, não trabalhava e não fazia uso de medicamentos ou hormônios.

Em 2019, o participante relatou ter 21 anos, natural de Pernambuco, reside em João Pessoa, sexo masculino, afirma ser homossexual, cursando o sexto do curso de nível superior, não trabalha e não faz uso de medicamentos ou hormônios.

Após esta etapa, as amostras da voz coletadas deste falante foram submetidas à avaliação perceptivo-auditiva por meio do roteiro VPAS- PB (CAMARGO e MADUREIRA, 2008) realizada por um juiz fonoaudiólogo com especialização em voz e experiência e vivência neste instrumento há 14 anos. Este juiz registrou seus julgamentos no próprio roteiro VPAS- PB impresso.

O VPAS-PB (CAMARGO e MADUREIRA, 2008) é uma adaptação do roteiro $V P A S$, de autoria do Laver et al (1981) que investiga a qualidade e dinâmica vocal partindo de uma abordagem fonética de classificação tradicional dos sons.

Além disso, o modelo é fundamentado em três princípios. Os dois primeiros referemse à relação entre ajustes: de compatibilidade, em que um ajuste exclui por antagonismo a execução do outro; de interdependência, em que um ajuste interfere na produção de outro (facilitando ou alterando a realização desse outro), estando ambos relacionados ao funcionamento interdependente dos movimentos musculares do trato vocal. O terceiro princípio refere se à relação entre ajustes e segmentos e denomina-se princípio de susceptibilidade. Neste, um segmento (vogal e consoante) pode ser suscetível à interferência de um ajuste; isto é, ele refere o grau de vulnerabilidade dos segmentos em relação aos ajustes, principalmente aqueles segmentos tidos como "chave" para a detecção dos eventos da qualidade vocal (MACKENZIE-BECK, 2005). Dessa forma, quando os ajustes apresentam características não compartilhadas pelo segmento, este último torna-se mais susceptível à influência dos primeiros.

Assim, o VPAS avalia perceptivo-auditivamente os ajustes laríngeos, supralaríngeos, de tensão muscular, bem como elementos de dinâmica vocal. Quanto aos ajustes laríngeos, o $V P A S$-PB identifica três tipos de elementos relacionados: ao modo de fonação (modal, falsete, crepitância/vocal fry, voz crepitante), fricção laríngea (escape de ar, voz soprosa), irregularidade laríngea (voz áspera). Em relação aos supralaríngeos: lábios (arredondados, estirados, labiodentalização, extensão diminuída e aumentada); mandíbula (fechada, aberta, protraída, extensão diminuída e aumentada); ponta de língua (avançada e recuada); corpo de língua (avançado, recuado, elevado, abaixado, extensão diminuída e aumentada); faringe (constrição e expansão); velofaringe (escape nasal audível, nasal e denasal); altura de laringe (elevada e abaixada). Para investigação da tensão muscular geral é avaliada a tensão do trato vocal (hiperfunção e hipofunção) e a tensão laríngea (hiperfunção e hipofunção). 
Em relação aos elementos de dinâmica vocal, os elementos prosódicos: pitch (habitual, extensão e variabilidade), loudness (habitual, extensão e variabilidade), tempo (continuidade, taxa de elocução) e outros elementos (suporte respiratório).

Vale salientar que o roteiro $V P A S$-PB não é um checklist, isto é, quando o juiz utiliza este roteiro, analisa na qualidade e dinâmica vocal o que é predominante quanto aos ajustes laríngeos, supralaríngeos, e de tensão muscular, bem como elementos prosódicos. Dessa forma, via de regra, ficam algumas lacunas do roteiro sem preencher, o que significa que aquele ajuste está neutro (em posição intermediária) naquele momento. Quando o ajuste é avaliado como não-neutro, utiliza-se uma escala de seis pontos, classificando o ajuste vocal como moderado (grau 1 a 3 ) ou extremo (grau 4 a 6).

Quanto à análise dos dados avaliados pelo juiz, estes foram descritos em um quadro baseado no roteiro $V P A S$ - PB, a seguir, e analisado qualitativamente.

\section{Resultados}

Os resultados da avaliação perceptivo-auditiva de qualidade e dinâmica vocal do sujeito homossexual, por meio do roteiro VPAS - PB, em dois períodos distintos, em 2016 e 2019 (Quadro 1). No ano de 2016, na qualidade vocal deste participante predominaram ajuste de mandíbula fechada, corpo de língua abaixado, expansão faríngea, laringe baixa, hiperfunção do trato vocal e voz modal, por outro lado, no ano de 2019 , houve o predomínio de ajuste de lábios estirados, mandíbula fechada, corpo de língua avançado, constrição faríngea, laringe elevada, hiperfunção do trato vocal, hiperfunção laríngea e falsete (Quadro 1).

Quanto aos ajustes de dinâmica vocal o participante do estudo de caso da presente pesquisa apresentou, no ano de 2016, um predomínio do ajuste de pitch habitual abaixado, pitch extensão diminuída e pitch variabilidade diminuída, entretanto, em 2019, houve predomínio de pitch habitual elevado, pitch extensão aumentada, pitch variabilidade aumentada e taxa de elocução rápida (Quadro 2).

Quadro 1- Julgamento perceptivo-auditivo da qualidade e dinâmica vocal do falante homossexual participante desta pesquisa em momentos diacrônicos (em 2016/ em 2019).

\begin{tabular}{|c|c|c|c|c|c|c|c|c|c|}
\hline \multirow[t]{3}{*}{ QUALIDADE VOCAL } & \multicolumn{2}{|c|}{ PRIMEIRA PASSADA } & \multicolumn{7}{|c|}{ SEGUNDA PASSADA } \\
\hline & \multirow{2}{*}{ Neutro } & \multirow{2}{*}{$\begin{array}{c}\text { Não } \\
\text { neutro }\end{array}$} & \multirow{2}{*}{ AJUSTE } & \multicolumn{3}{|c|}{ Moderado } & \multicolumn{3}{|c|}{ Extremo } \\
\hline & & & & 1 & 2 & 3 & 4 & 5 & 6 \\
\hline \multicolumn{10}{|c|}{ A. ELEMENTOS DO TRATO VOCAL } \\
\hline \multirow{5}{*}{ 1.Lábios } & & & Arredondados/protraídos & & & & & & \\
\hline & & & Estirados & & & $\boldsymbol{\Delta}$ & & & \\
\hline & & & Labiodentalização & & & & & & \\
\hline & & & Extensão diminuída & & & & & & \\
\hline & & & Extensão aumentada & & & & & & \\
\hline \multirow{5}{*}{ 2. Mandíbula } & & & Fechada & $\Delta$ & & $\mathbf{X}$ & & & \\
\hline & & & Aberta & & & & & & \\
\hline & & & Protraída & & & & & & \\
\hline & & & Extensão diminuída & & & & & & \\
\hline & & & Extensão aumentada & & & & & & \\
\hline
\end{tabular}


Volume 14 - Número 1 - mai/ago de 2019

\begin{tabular}{|c|c|c|c|c|c|c|c|c|c|}
\hline \multirow{2}{*}{ 3.Língua ponta/lâmina } & & \multicolumn{2}{|c|}{ Avançada } & & & & & & \\
\hline & & \multicolumn{2}{|c|}{ Recuada } & & & & & & \\
\hline \multirow{6}{*}{ 4. Corpo de língua } & & \multicolumn{2}{|c|}{ Avançado } & $\boldsymbol{\Delta}(\mathrm{i})$ & & & & & \\
\hline & & \multicolumn{2}{|c|}{ Recuado } & & & & & & \\
\hline & & \multicolumn{2}{|c|}{ Elevado } & & & & & & \\
\hline & & \multicolumn{2}{|c|}{ Abaixado } & $\mathbf{X}$ & & & & & \\
\hline & & \multicolumn{2}{|c|}{ Extensão diminuída } & & & & & & \\
\hline & & \multicolumn{2}{|c|}{ Extensão aumentada } & & & & & & \\
\hline \multirow{2}{*}{ 5.Faringe } & & \multicolumn{2}{|c|}{ Constrição } & $\boldsymbol{\Delta}$ & & & & & \\
\hline & & \multicolumn{2}{|c|}{ Expansão } & $\mathbf{X}$ & & & & & \\
\hline \multirow{3}{*}{ 6.Velofaringe } & & \multicolumn{2}{|c|}{ Escape nasal audível } & & & & & & \\
\hline & & \multicolumn{2}{|c|}{ Nasal } & & & & & & \\
\hline & & \multicolumn{2}{|c|}{ Denasal } & & & & & & \\
\hline \multirow{2}{*}{ 7. Altura de laringe } & & \multicolumn{2}{|c|}{ Elevada } & & & & $\boldsymbol{\Delta}$ & & \\
\hline & & \multicolumn{2}{|c|}{ Abaixada } & & $\mathbf{X}$ & & & & \\
\hline \multicolumn{10}{|c|}{ B. TENSÃO MUSCULAR GERAL } \\
\hline \multirow{2}{*}{ 8. Tensão do trato vocal } & & \multicolumn{2}{|c|}{ Hiperfunção } & $\mathbf{X}(\mathbf{i})$ & & $\Delta$ & & & \\
\hline & & \multicolumn{2}{|c|}{ Hipofunção } & & & & & & \\
\hline \multirow{2}{*}{ 9. Tensão laríngea } & & \multicolumn{2}{|c|}{ Hiperfunção } & & & $\Delta$ & & & \\
\hline & & \multicolumn{2}{|c|}{ Hipofunção } & & & & & & \\
\hline \multicolumn{10}{|c|}{ C. ELEMENTOS FONATÓRIOS } \\
\hline & \multirow{3}{*}{ AJUSTE } & $\operatorname{Pr}$ & & & Gra & us d & es & ala & \\
\hline & & & Não & Mo & der: & & & tre & \\
\hline & & reutro & Neutro & 1 & 2 & 3 & 4 & 5 & 6 \\
\hline & Modal & $\mathbf{X}$ & & & & & & & \\
\hline 10 Modo de fonac̃̃ & Falsete & & $\boldsymbol{\Delta}(\mathrm{i})$ & & & & & & \\
\hline & Crepitância/ vocal fry & & & & & & & & \\
\hline & Voz crepitante & & & & & & & & \\
\hline 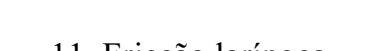 & Escape de ar & & & & & & & & \\
\hline & Voz soprosa & & & & & & & & \\
\hline 12.Irregularidade laríngea & Voz áspera & & & & & & & & \\
\hline
\end{tabular}

Legenda: $\Delta$ Depois dos 3 anos (2019); $\quad \mathbf{X}$ Antes dos 3 anos (2016)

(i) Ajuste intermitente 
Quadro 2- Julgamento perceptivo-auditivo da dinâmica vocal do sujeito participante desta pesquisa em momentos diacrônicos.

\begin{tabular}{|c|c|c|c|c|c|c|c|c|c|}
\hline \multirow{2}{*}{\multicolumn{2}{|c|}{ DINÂMICA VOCAL }} & \multirow{2}{*}{ Neutro } & \multirow{2}{*}{ AJUSTE } & \multicolumn{3}{|c|}{ Moderado } & \multicolumn{3}{|c|}{ Extremo } \\
\hline & & & & 1 & 2 & 3 & 4 & 5 & 6 \\
\hline \multicolumn{10}{|c|}{ D. ELEMENTOS PROSÓDICOS } \\
\hline \multirow{6}{*}{ 13.Pitch (f0) } & \multirow{2}{*}{ Habitual } & & Elevado & & & & $\Delta$ & & \\
\hline & & & Abaixado & $\mathbf{X}$ & & & & & \\
\hline & \multirow{2}{*}{ Extensão } & & Diminuída & $\mathbf{X}$ & & & & & \\
\hline & & & Aumentada & & & & $\Delta$ & & \\
\hline & \multirow{2}{*}{ Variabilidade } & & Diminuída & $\mathbf{X}$ & & & & & \\
\hline & & & Aumentada & & & & $\mathbf{\Delta}$ & & \\
\hline \multirow{6}{*}{$\begin{array}{l}\text { 14.Loudness } \\
\text { (intensidade) }\end{array}$} & \multirow{2}{*}{ Habitual } & & Aumentado & & & & & & \\
\hline & & & Diminuído & & & & & & \\
\hline & \multirow{2}{*}{ Extensão } & & Diminuída & & & & & & \\
\hline & & & Aumentada & & & & & & \\
\hline & \multirow{2}{*}{ Variabilidade } & & Diminuída & & & & & & \\
\hline & & & Aumentada & & & & & & \\
\hline \multicolumn{10}{|c|}{ 15. Tempo } \\
\hline Continu & & & Interrompida & & & & & & \\
\hline \multirow{2}{*}{\multicolumn{2}{|c|}{ Taxa de elocução }} & & Rápida & & & $\Delta$ & & & \\
\hline & & & Lenta & & & & & & \\
\hline \multicolumn{10}{|c|}{ 16.OUTROS ELEMENTOS } \\
\hline \multirow{2}{*}{\multicolumn{3}{|c|}{ Suporte respiratório }} & Adequado & & & & & & \\
\hline & & & Inadequado & & & & & & \\
\hline
\end{tabular}

Legenda: $\mathbf{\Delta}$ Depois dos 3 anos (2019); $\mathbf{X}$ Antes dos 3 anos (2016)

(i) Ajuste intermitente

\section{Discussão}

O escopo deste trabalho reflete uma análise da variação de qualidade vocal de um falante homossexual em dois momentos distintos, separados por um intervalo de tempo de três anos. Esse falante apresentou qualidades vocais distintas nos dois momentos, antes e depois de assumir sua condição homossexual, o que motivou a investigação de tais observações. A análise foi desenvolvida seguindo a perspectiva teórica da Descrição Fonética de Qualidade Vocal (LAVER, 1980), pela qual buscou-se compreender o papel dos ajustes realizados pelos articuladores na qualidade e dinâmica vocal desse falante, além de detectar que características o aproximam de sua comunidade de práticas linguísticas.

O modelo Fonético de Descrição da Qualidade Vocal consiste numa abordagem analítica, de caráter científico que define o ajuste (setting) como unidade de análise (LAVER, 1980). O $V P A S$-PB é de extrema utilidade para a amostra desta pesquisa por proporcionar o mapeamento dos ajustes de qualidade e os aspectos de dinâmica vocal (recursos prosódicos), além de permitir ao fonoaudiólogo construir um raciocínio claro sobre as estratégias e técnicas vocais utilizadas por este indivíduo para melhoria da qualidade vocal e da expressividade da fala, e consequentemente da qualidade de vida deste sujeito (ALBANO, 2001; LIMA-SILVA, 2012; PEREIRA et al, 2017).

As discussões desta pesquisa centram-se na presença de ajustes de qualidade e dinâmica vocal em um falante homossexual comparando dois momentos da sua vida: no primeiro momento, antes de se considerar homossexual; e no segundo momento, no qual já se considerava homossexual.

No primeiro momento o falante homossexual apresentou ajustes que são compatíveis com falantes do sexo masculino como laringe baixa, corpo de língua abaixado, mandíbula 
fechada (LIMA et al., 2007). Em contrapartida, no segundo momento verificou-se ajustes compatíveis com falantes do sexo feminino como laringe elevada, constrição faríngea e hiperfunção laríngea (LIMA et al., 2007; LIMA-SILVA, 2012).

A maioria das mudanças dos ajustes identificados após o intervalo de três anos (tais como constrição faríngea, laringe elevada, lábios estirados e hiperfunção laríngea) geram mudanças nos ajustes do trato vocal, tendo como consequência a elevação do pitch habitual, o que foi compatível com os achados encontrados na presente pesquisa (LAVER, 1980).

Além disso, quanto ao ponto de vista da Sociolinguística as mudanças apresentadas levam a alguns indícios sobre as novas atribuições de significados sociais realizadas pelo falante homossexual investigado, que passa a considerar sua voz como instrumento não apenas fisiológico de comunicação, mas como um molde de sua persona, da comunidade ao qual está inserido (ECKERT, 2012).

A presença de hiperfunção laríngea e da laringe elevada no presente estudo pode causar fechamento glótico vigoroso e modificar o modo de vibração das pregas vocais causando mudanças no desempenho a nível fonatório, bem como nos aspectos de dinâmica vocal (LAVER, 1980; PINHO; PONTES, 2008; LIMA-SILVA, 2012). O princípio de compatibilidade pode auxiliar a justificar a presença de ajustes de laringe elevada e hiperfunção laríngea alertando para a possibilidade de predisposição para distúrbios de voz, neste falante (LAVER, 1980; LIMA-SILVA, 2012; QUEIROZ, 2012).

Quanto aos aspectos de dinâmica vocal, os elementos que compõem o item prosódia no roteiro VPAS-PB como pitch, loudness e taxa de elocução, normalmente utilizados como funções contempladoras das dimensões extralinguísticas, linguísticas e paralinguísticas, nível em que se encontra a expressão vocal das emoções (LAVER, 1994, MADUREIRA, 2016), mostram que a diminuição da extensão do trato vocal ocasiona a sobrecarga dos mecanismos fonatórios e, dessa forma, causa interferências nos aspectos de dinâmica vocal (LAVER, 2000; PINHO; PONTES, 2008; FERNANDES, 2011; LIMA-SILVA, 2012).

No momento após assumir-se homossexual, ocorreu o aumento dos ajustes de taxa de elocução rápida, que pode afetar diretamente a qualidade e expressividade de fala, interferindo também na prosódia (YILDIRIM, et al. 2004; RODRIGUES, 2007; MADUREIRA, 2016). As informações contidas nas análises realizadas através do roteiro $V P A S$ são de extrema importância não só para identificação de quadros de distúrbio de voz, mas também para descrição da prosódia (expressividade da fala). Ao adotar estratégias de modificações do trato vocal, este sujeito pode realizar diversas mudanças nos aspectos de dinâmica vocal para se adequar ao tipo de mensagem ou sentimento a serem expressos, evidenciando a plasticidade do trato vocal (MADUREIRA, 2010; MARQUEZIN, et al. 2015).

Os dados mostraram uma considerável mudança nos padrões de qualidade e dinâmica vocal do sujeito analisado. Tendo como premissas a teoria da Sociolinguística, pode-se perceber que essas mudanças (variações, no termo mais exato) representam um fenômeno cujo conceito é bastante caro à Sociolinguística: o conceito de variação diacrônica, que revela as variações de determinadas marcas linguísticas ao longo do tempo cronológico.

Considerando os ajustes realizados pelo falante analisado no segundo momento, tais como a constrição faríngea, a laringe elevada e a hiperfunção do trato vocal, percebe-se considerável mudança de ajustes ao longo do tempo corroborando o conceito de variação diacrônica. Ao observar tais recursos utilizados nos ajustes desse falante, percebe-se ainda que tais mudanças atestam os fatores envolvidos na produção linguística, que podem ser de ordem linguística e extralinguística (WEINREICH; LABOV; HERZOG, 2006).

Ao considerar o fato de o falante ter assumido a homossexualidade no segundo momento de amostra, pode-se pensar que as mudanças demonstradas na sua qualidade e 
dinâmica vocal refletem ainda fatores de natureza social e cultural, representando uma covariação sistemática entre o material linguístico e as diversas conjunturas socioculturais.

Diante disso, é válido ressaltar que, por conceber, a sua condição homossexual e o modo como a sociedade a enxerga (por vezes com preconceito e discriminação), o falante utiliza-se de sua qualidade e dinâmica vocal como maneira de atestar a consciência de sua inserção nessa condição e nessa sociedade. Obviamente, pensar nessa inserção conecta-se ao pensamento que de os significados sociais impetrados às suas variações de voz refletem a concepção de que esses significados não podem ser vistos de forma isolada dessa realidade social.

Ainda pensando nessa mudança de qualidade e dinâmica de voz, nota-se uma nova construção identitária desse sujeito, cujas reflexões permeiam crenças em novos valores sociais e culturais e trazem à tona a transitoriedade da identidade, abordada por Hall (2006) como construções que variam ao longo do tempo. Além disso, o autor é categórico ao resgatar a noção de identidade de Bauman (2005), mas acrescentando à sua contribuição a noção de identidades culturais, cujos aspectos mais relevantes advém de nosso pertencimento a culturas étnicas, raciais, linguísticas.

Destarte, é possível observar que as mudanças apresentadas pelo sujeito permitem visualizar um processo de variação que implica inferir acerca de sua interligação com uma rede de conexões contextuais e identitárias.

Por isso, volta-se à perspectiva de que os estudos fonéticos, em suas mais variadas direções, podem se ligar com profundidade aos mais diversos campos de estudos como a Fonoaudiologia, uma vez que a voz é tão complexa quanto a fala. Por isso, considera-se que a integração de perspectivas entre voz e fala deve crescer e se solidificar nas duas áreas, da Sociolinguística e da Fonoaudiologia. Na Sociolinguística a fim de que se possa continuar a atestar que o "caos linguístico" pode ser (e é) explicado, descrito e desmitificado; e na Fonoaudiologia, especificamente na área da voz, na tentativa de resgatar a abordagem integrada do sinal sonoro (sinal vocal como parte integrante do sinal da fala).

Sugere-se para futuros estudos a complementariedade de outros instrumentos de análise, por exemplo, a análise acústica e fisiológica para verificar a acurácia desses dados, bem como ampliar a amostra.

\section{Conclusão}

Conclui-se que no primeiro momento da coleta, antes de assumir-se homossexual (em 2016) o participante apresentou os seguintes ajustes de trato vocal: mandíbula fechada (grau 03); corpo de língua abaixada (grau 01); expansão faríngea (grau 01); laringe abaixada (grau 02); hiperfunção do trato vocal (grau 01); modal pitch habitual abaixado (grau 01); e por fim extensão e variabilidade de pitch diminuído (grau 01)

No segundo momento, após assumir-se homossexual (em 2019) o participante apresentou os seguintes ajustes de trato vocal: mandíbula fechada (grau 03); corpo de língua avançado (grau 01); constrição faríngea (grau 01); laringe elevada (grau 04); hiperfunção do trato vocal (grau 03); hiperfunção laríngea (grau 03); falsete (i), pitch elevado grau (04); extensão e variabilidade de pitch aumentada (grau 04); e por fim taxa de elocução rápida (grau 03).

Nessa investigação foi possível traçar o perfil vocal do falante homossexual em momentos distintos identificando ajustes marcantes na fala deste sujeito, bem como as relações sociais e de atribuições de significados estabelecidos por esses ajustes. É importante ressaltar que os dados obtidos não generalizam o perfil vocal de todos os falantes homossexuais, considerando as diferentes configurações do aparelho fonador e das visões de 
cada falante, uma vez que este estudo foi realizado apenas com um falante e não com toda uma comunidade homossexual.

Nota-se que os ajustes apresentados no segundo momento de análise representam facetas sociais claramente marcadas pela ligação do falante às suas comunidades de práticas após assumir-se homossexual, demonstrando que os significados sociais atribuídos por esse falante mudaram de acordo com a sua percepção da sua sexualidade, como por exemplo, a luta pela aceitação da sociedade e a resistência da comunidade homossexual. Além disso, a mudança da qualidade vocal e dos aspectos de dinâmica vocal apresenta uma das características marcantes da variação linguística: a variação diacrônica, o que corrobora um traço integrador entre Sociolinguística e Fonética.

\section{Referências}

ALBANO, E. C. O gesto e suas bordas: esboço de fonologia acústico-articulatória do português brasileiro. Campinas: Mercado das Letras, 2001.

BAUMAN, Z. Identidade: entrevista a Benedetto Vecchi. Rio de Janeiro: Jorge Zahar, 2005

CAMARGO, Z. A.; MADUREIRA, S. Avaliação vocal sob a perspectiva fonética: investigação preliminar. Distúrbios da Comunicação, v. 20, n. 1, 2008.

CEZARIO, M. M.; VOTRE, S. Sociolinguística. In: MARTELOTTA, M. (org.). Manual de linguística. São Paulo: Contexto, 2011. p. 141-155.

CRISPIM, K.; SPINA, D. Post-stressed Syllables in Brazilian Portuguese as Markers. Proceedings of ICPhS, San Francisco, v. 2, n.1, 1999.

ECKERT, P. Variation, convention and social meaning. Paper Presented at the Annual Meeting of the Linguistic Society of America. Oakland CA. Jan. 7, 2005.

HALL, S. A identidade cultural na pós-modernidade (11 a . Edição). São Paulo: DP\&A, 2006. LABOV, W. Where does the Linguistic variable stop? A response to Beatriz Lavandera. In: Sociolinguistic Working Papers, 1978.

LAVER, J. et al. A perceptual protocol for the analysis of vocal profiles. Edinburg University Department of Linguistics Work in Progress, p. 139-55, 1981.

LAVER, J. Phonetic evaluation of voice quality. In: KENT, R. D.; BALL, M. J. Voice quality measurement. San Diego: Singular Publishing Group, p. 37-48, 2000.

LAVER, J. The phonetic description of voice quality. New York: Cambridge University Press; 1980.

LAVER J, MACKENZIE-BECK J. Vocal Profile Analysis Scheme - VPAS. Edinburgh, Apostila do Curso do VPAS - Queen Margareth University College - QMUC, Speech Science Research Centre, 2007.

LIMA, M. F.B. et al. Qualidade vocal e formantes das vogais de falantes adultos da cidade de João Pessoa Voice quality and vowel formants of adult subjects from João Pessoa city. Revista CEFAC, v. 9, n. 1, p. 99-109, 2007.

LIMA-SILVA, M. F. B. et al. Distúrbio de voz em professores: autorreferência, avaliação perceptiva da voz e das pregas vocais. Rev. Soc. Bras. Fonoaudiol, v. 17, n. 4, p. 391-397, 2012.

MACKENZIE-BECK, J. Perceptual analysis of voice quality: the place of vocal profile analysis. A Figure of Speech. A Festschrift for John Laver, p. 285-322, 2005.

MADUREIRA S. O sentido do som [Tese de doutorado]. São Paulo: Pontifícia Universidade Católica de São Paulo; 1992.

MADUREIRA, S. Reciting a sonnet: production strategies and perceptual effects. In: Proceedings of the Speech Prosody, 2008 Conference. p. 697-700, 2008.

MADUREIRA, S.; CAMARGO, Z. Exploring sound symbolism in the investigation of speech expressivity. In: Proceedings of the third ISCA Tutorial and research workshop on 
Experimental Linguistcs; 2010; Athens, Greece. Athens: International Speech Communication Association ISCA, p. 105-8, 2010.

MADUREIRA, Sandra; CAMARGO, Zuleica Antonia de. Exploring sound symbolism in the investigation of speech expressivity. In: Third ISCA Workshop on Experimental Linguistics. 2010.

MARQUEZIN, D. M. et al. Expressividade da fala de executivos: análise de aspectos perceptivos e acústicos da dinâmica vocal. In: CoDAS. p. 160-9, 2015.

MEDEIROS, C. M. A. Aspectos fonéticos perceptivos auditivos e acústicos da qualidade vocal de teleoperadores de emergência antes e após jornada de trabalho. Dissertação de Mestrado (Pós-Graduação em Linguística) - Universidade Federal da Paraíba, 2019.

NASCIMENTO, I. C.; A percepção das vogais médias pretônicas por estudantes do ensino fundamental II e o ensino de língua portuguesa. Monografia (Graduação em Letras / Língua portuguesa) - Universidade Federal da Paraíba, 2017.

PEREIRA, E. C. et al. Análises perceptivo-auditiva e acústica das vozes de crianças infectadas pelo HIV. CEP, v. 84400, p. 000, 2017.

PINHO, S. M. R.; PONTES, P. Músculos intrínsecos da laringe e dinâmica vocal. Rio de Janeiro: Revinter, 2008.

QUEIROZ, R. Qualidade vocal: análise acústica de ajuste fonatório e de tensão laríngea. Dissertação de Mestrado, Pontifícia Universidade Católica de São Paulo, 2012.

RODRIGUES, L.L. Aquisição dos róticos em crianças com queixa fonoaudiológica. Dissertação em Linguística. Campinas: UNICAMP, 2007.

YILDIRIM S., et al. Study of acoustic correlates associate with emotional speech. Meeting of the ASA, San Diego, 2004.

Recebido em 18/05/19

Aceito em 30/09/19 